\title{
Multispectral MR to X-Ray Registration of Vertebral Bodies by Generating CT-Like Data
}

\author{
Everine B. van de Kraats ${ }^{1}$, Graeme P. Penney ${ }^{2}$, Theo van Walsum ${ }^{3}$, \\ and Wiro J. Niessen ${ }^{3}$ \\ 1 Image Sciences Institute, University Medical Center Utrecht, The Netherlands \\ everine@isi.uu.nl \\ 2 Dept. of Computer Science, University College London \\ 3 Biomedical Imaging Group, Erasmus MC, University Medical Center Rotterdam
}

\begin{abstract}
A new method for MR to X-ray registration is presented. Based on training data, consisting of registered multispectral MR and CT data, a function is defined that maps multispectral MR data to CTlike data. For new subjects for which multispectral MR data have been acquired, the mapping function is used to generate a corresponding CTlike dataset. The CT-like image is subsequently used for registration to $\mathrm{X}$-ray data, using gradient-based registration. Preliminary experiments indicate that MR to X-ray registration using this method is more accurate and has a larger capture range than gradient-based registration applied directly to MR data.
\end{abstract}

\section{Introduction}

In diagnosis and therapy planning, volumetric magnetic resonance (MR) images and computed tomography (CT) datasets are often used. However, in many interventions, intraoperative imaging is limited to 2D; in intravascular procedures and in orthopedic interventions, for example, usually only X-ray projection images are available. In order to relate these images to preoperative data so as to provide $3 \mathrm{D}$ insight during the intervention, 2D-3D registration is required.

Several 2D-3D registration methods have been reported in literature. Most algorithms have been designed for CT to X-ray registration. Since both these imaging modalities rely on X-rays, this can be exploited in designing 2D-3D registration methods. MR to X-ray registration is much more challenging, owing to the different underlying contrast mechanism. Several papers have appeared that address registration of magnetic resonance angiography (MRA) to digital subtraction angiography (DSA) images (among others [1, 2, 3]) and little has been done for MR to X-ray registration of bone [4,5]. Rohlfing [4] compares intensity distributions along all rays to the corresponding pixel intensities of the real projection images by means of a probabilistic extension of histogram-based similarity measures, and Tomaževič [5] optimizes alignment of the gradients in $2 \mathrm{D}$ and $3 \mathrm{D}$ images. The main difficulty in $\mathrm{MR}$ to $\mathrm{X}$-ray registration for bone applications is that bone tissue is not well depicted in MR images. This makes direct application of gradient-based methods, that rely on the bone edge or 
surface, or intensity-based methods, that assume a relation between the image intensities, difficult.

In this paper, a new approach for MR to X-ray registration is proposed. It is investigated whether multispectral MR data can be used to construct a CTlike image, containing sufficient information for registration to X-ray projection images. Hereto, a mapping function is defined, based on training data, which maps multispectral MR data obtained with a standardized acquisition protocol, into a simulated CT dataset. This is not straightforward, as MR imaging is not quantitative and MR intensities are affected by position dependent variations in coil and scanner sensitivity.

In the following, we first introduce the method to construct a CT-like image from multispectral MR data. Next, 2D-3D registration based on the constructed CT-like data is described and the datasets that are acquired for training and evaluation are specified. Subsequently, the experiments are described that were conducted to assess whether in controlled conditions MR intensities can be assumed to be similar across subjects for our purpose, or whether intensity remapping is required. Finally, the 2D-3D registration experiments with the CT-like datasets are detailed and the results are presented and discussed.

\section{Method}

\subsection{Training Multispectral MR to CT Mapping}

The general idea is to investigate whether a function can be proposed that maps multispectral MR datasets that are acquired with a standardized protocol to CTlike data. This mapping function can depend on MR image intensities, image features, and spatial information. For a proof of concept, we use a straightforward approach, in which only the image intensities of three different MR scans are used.

In the training stage, registered multispectral MR and CT datasets are used. From these datasets, a look-up table (LUT) is constructed relating MR-value triplets to CT values. Hereto, intensities in each of the MR images are divided in $n$ bins each:

$$
\mathrm{BIN}_{\mathrm{i}}(\mathbf{x})=\left\lfloor\frac{I_{i}(\mathbf{x})-\min (I)}{(\max (I)-\min (I)) / n}\right\rfloor,
$$

where

$$
\begin{aligned}
I_{i}(\mathbf{x}) & =\text { intensity of voxel } \mathbf{x} \text { in MR image } i \\
\min (I) / \max (I) & =\text { minimum } / \text { maximum intensity over all MR images. }
\end{aligned}
$$

In this preliminary experiment, 64 evenly distributed bins were used. A region of interest is determined containing the specific anatomy that is trained. In our case this is a specific level of the spine (or several spine levels).

The simulated $\mathrm{CT}$ value in a certain region corresponding to a specific MRvalue triplet, is determined using a mapping function $f$ :

$$
\operatorname{LUT}(u, v, w)=f\left(\left\{\mathrm{CT}(\mathbf{x}) \mid \mathbf{x} \in S_{u, v, w}^{r}\right\}\right),
$$


where

$S_{u, v, w}^{r}=$ set of voxels $\mathbf{x}$ in region $r$ where $\mathrm{BIN}_{1}(\mathbf{x})=u, \mathrm{BIN}_{2}(\mathbf{x})=v$ and $\mathrm{BIN}_{3}(\mathbf{x})=w$,

$f: \mathbb{Z}^{q} \rightarrow \mathbb{R}$ is function that maps the set of $\mathrm{CT}$ values corresponding to a certain MR-value triplet into a single estimated CT value.

We chose the median function for $f$. The mapping functions can easily be extended, e.g. by incorporating feature information, information over larger neighborhoods, or spatial information (e.g. an atlas). In addition, binning techniques more effective than uniform binning may be useful.

\subsection{D-3D Registration Based on a CT-Like Dataset}

For a new subject for which a multispectral MR dataset is available, a trained mapping function as defined in the previous section can be used to generate a CT-like image. This image is subsequently used in 2D-3D registration. Since the CT-like image consists of values that are similar to CT values, intensitybased registration methods as well as feature-based or gradient-based registration methods can be applied.

In this preliminary experiment, we considered a gradient-based 2D-3D registration approach [5, which was previously used for MR to X-ray registration of the spine. Registration to two X-ray images was considered, as one X-ray image is not sufficient to assess the position in three dimensions.

For validation of the 2D-3D registration we applied the framework that was introduced in [6]. Using an accurate reference standard the performance of the method was evaluated by reporting the capture range and final mean TRE (mTRE) of successful registrations within the capture range.

\section{$3 \quad$ Experiments}

\subsection{Data Acquisition}

First, multispectral MR datasets of six healthy subjects (one male and five females with ages ranging from 21 to 54) were acquired using a standardized protocol (see Table 1) with a 1.5-T MR scanner (GyroScan NT, Philips Medical Systems, Best, The Netherlands). Two of the subjects were scanned in two MR scanners, with the same specifications but at different locations, to assess interscanner variability. Histograms of all MR images of the same anatomic regions were compared qualitatively, to determine whether intensity remapping of the MR data would be required for training the LUT and applying it to new cases.

Second, multispectral MR (using the same protocol as above), CT, and 3DRX datasets with corresponding X-ray images were acquired from two fresh postmortem subjects (females aged 102 and 77). CT images were acquired on a clinical multi-slice CT scanner (MX8000, IDT16, Philips Medical Systems). CT and MR datasets were registered using maximization of mutual information. 
Table 1. Specifications of the acquired scans

\begin{tabular}{|c|c|}
\hline Protocol name & Specifications \\
\hline $\begin{array}{l}\text { Balanced Fast Field Echo } \\
\text { (BFFE) }\end{array}$ & $\begin{array}{l}\text { TR } 5.29 \text {, TE } 1.80, \text { NA } 8.0 \text {, slice thickness } 3.0 \mathrm{~mm} \text {, } \\
\text { slice spacing } 1.5 \mathrm{~mm} \text {, pixel spacing } 0.5 / 0.5 \mathrm{~mm} \text {, flip } \\
\text { angle } 50^{\circ}, 512 \times 512 \times 70\end{array}$ \\
\hline $\begin{array}{l}\text { Balanced Fast Field Echo } \\
\text { with water selection } \\
\text { (BFFE_WS) }\end{array}$ & $\begin{array}{l}\text { TR } 6.21 \text {, TE } 3.10 \text {, NA } 8.0 \text {, slice thickness } 3.0 \mathrm{~mm} \text {, } \\
\text { slice spacing } 1.5 \mathrm{~mm} \text {, pixel spacing } 0.5 / 0.5 \mathrm{~mm} \text {, flip } \\
\text { angle } 50^{\circ}, 512 \times 512 \times 70\end{array}$ \\
\hline $\begin{array}{l}\text { Balanced Fast Field Echo } \\
\text { with fat selection } \\
\text { (BFFE_FS) }\end{array}$ & $\begin{array}{l}\text { TR } 6.21 \text {, TE } 3.10 \text {, NA } 8.0 \text {, slice thickness } 3.0 \mathrm{~mm} \text {, } \\
\text { slice spacing } 1.5 \mathrm{~mm} \text {, pixel spacing } 0.5 / 0.5 \mathrm{~mm} \text {, flip } \\
\text { angle } 50^{\circ}, 512 \times 512 \times 70\end{array}$ \\
\hline CT & $0.35 \times 0.35 \times 0.5 \mathrm{~mm}^{3}$, slice thickness $1.0 \mathrm{~mm}$ \\
\hline $3 \mathrm{DRX}$ & $0.43 \times 0.43 \times 0.43 \mathrm{~mm}^{3}, 256 \times 256 \times 256$ \\
\hline 200 X-ray projections & $0.44 \times 0.44 \mathrm{~mm}^{2}, 512 \times 512,25 \mathrm{~cm} \mathrm{II}$ \\
\hline
\end{tabular}

These registered datasets were used to train the LUT. 3DRX data and X-ray images were acquired using a 3D C-arm system (Integris BV5000, Philips Medical Systems). Table 1 describes the details of the acquired scans.

The geometrical relation between X-ray images and the 3DRX volume was known, because the 3DRX volume is reconstructed from the X-ray projections. This serves as a reference standard for 3DRX to X-ray registration, as proposed by van de Kraats et al. [6]. The CT and MR data were registered to the 3DRX data using maximization of mutual information. As a consequence, the relationship between the X-ray images and the CT and MR data was indirectly derived, which provided the reference standard for MR to X-ray and CT to X-ray registration.

\subsection{MR Histogram Inspection}

For the six healthy subjects it was investigated whether intensity remapping was required to relate image intensities between subjects. We also qualitatively assessed the relation between MR intensities of healthy subjects and postmortem subjects.

The comparison of MR intensities (across different scans or patients) was performed using the MR intensities that result directly from the Fourier transformed signal intensities, to circumvent the linear transformations that are normally applied in postprocessing steps by MR scanners. We determined regions of interest around the vertebra for each of the subjects and for all the scans. Since the region of interest affects the histogram, it was crucial that similar regions of interest were chosen. In this experiment, a narrow region of interest around vertebral body L3 was taken.

The resulting histograms indicated that the MR intensities were indeed comparable and that age, sex, or scanner did not influence the histograms profoundly. The peaks in the histograms per MR protocol occurred at similar intensities. For the BFFE scan the mean and standard deviation of the position of the peaks was 

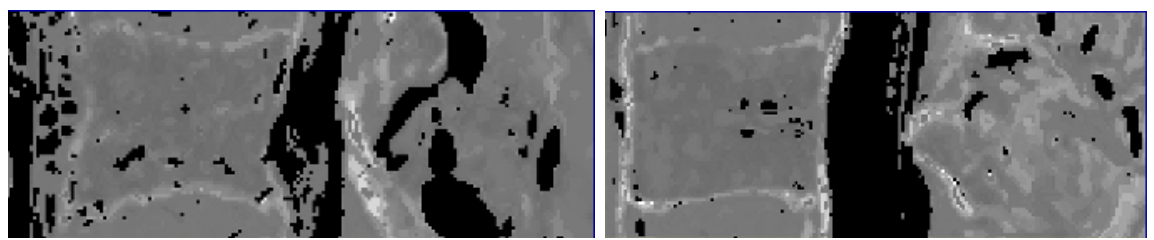

Fig. 1. Sagittal plane through center of vertebra and spinal canal of CT-like images of two healthy subjects constructed using a LUT trained on a postmortem subject (SA-L1)

$19.1 \pm 3.5$, for BFFE_WS this was 47.6 \pm 4.9 , and for BFFE_FS this was $7.7 \pm 1.9$. Thus it was expected that application of a LUT trained on any one of these multispectral MR datasets would result in similar CT-like images. A logical step was to verify this by using the trained LUT obtained from the postmortem scans. However, from a similar analysis, it seemed that the histograms of fresh postmortem subjects did not correspond well with healthy subjects. Since the availability of MR, CT, and 3DRX data of the same patient is rare, we had to rely on the (fresh) postmortem data to relate MR and CT intensities. To overcome the intensity correspondence problem, we linearly rescaled the images of the postmortem subjects in such a way that the histogram peaks roughly overlapped with the peaks of the healthy subjects before these were used to train the LUT. This was done in an ad hoc manner; for better histogram overlap more sophisticated techniques can be used. The resulting CT-like images, for which one slice is shown for two subjects in Figure 1 could not be evaluated quantitatively as corresponding CT and 3DRX scans were not acquired of volunteers. The dark areas in the images correspond to locations for which no or insufficient data was present in the training set. These locations can be filled, e.g. using gray value dilation or inpainting. The dark areas mainly occur in the spinal canal. This is possibly due to differences between in vivo and in vitro spinal fluid. However, the resulting CT-like images look promising as edges are present at positions where a bone edge is expected.

\section{$3.3 \quad 2 D-3 D$ Registration}

Experiments were conducted to determine the performance of 2D-3D registration using the simulated CT-like datasets in the postmortem study. An important aspect was to decide on what dataset and region to train the LUT. Whereas we hypothesize that the LUT will improve with a larger training set, in our first experiments only two datasets were available: a postmortem female aged 102 (SA) and a postmortem female aged 77 (SB). These differred substantially in quality as SA had a very deformed spine with hardly any disc spaces and a high degree of osteoporosis. We therefore did not use this dataset for training, but for evaluation; vertebra L3 (SA-L3) was used for this purpose. To determine the performance as a function of the training data, we trained on SA-L3, SB-L3, SB-L1 and SB-L1,L3. 
To assess the performance of $2 \mathrm{D}-3 \mathrm{D}$ registration using the CT-like image in comparison to registrations using 3DRX, CT, and original MR data, we also evaluated the performance of 2D-3D registration using corresponding regions of interest in the 3DRX, CT, resampled CT image ('trans CT', which resembles the resolution of the MR images, $\left.1.5 \times 0.5 \times 0.5 \mathrm{~mm}^{3}\right)$, and the original $\mathrm{MR}$ scan $(\mathrm{BFFE})$. For each of the corresponding vertebra volumes, the same two fluoroscopic images were used for registration. These were approximately at a $90^{\circ}$ angle.

For all the datasets, 200 registrations were performed, using the same offsets from the gold standard, which ranged from 0 to $10 \mathrm{~mm}$ mTRE with 20 starting positions per $1 \mathrm{~mm}$. The 100 and 90 percent capture ranges, where success was defined as an error smaller than $2 \mathrm{~mm}$ mTRE, are reported together with the mean accuracy for successful registrations.

\section{Results and Discussion}

Figure 2 shows corresponding slices of MR BFFE data, transformed CT data (registered to MR BFFE data), and four generated CT-like datasets (acquired using LUTs trained on different vertebrae) for vertebra L3 of subject A. Whereas it can clearly be seen that when training on the vertebra itself, the generated CT-like image resembles the CT scan best (Figure 2k), in all generated CT-like data edges are present at bone surfaces. There were only a few nontrained MRintensity triplets (holes in the CT-like datasets), and we thus expect that the unfilled locations have minimal effect on the registration result.

Table 2 lists the 2D-3D registration results. The $100 \%$ and $90 \%$ capture ranges (where success was defined as a final mTRE less than $2 \mathrm{~mm}$ ) are reported along with the average error of successful registrations within these ranges. As expected, registration of the 3DRX image to the two X-ray images had the highest $100 \% / 90 \%$ capture range $(5 / 6 \mathrm{~mm}$ start $\mathrm{mTRE})$ with an mTRE of $0.30 \mathrm{~mm}$, followed by registration of the original CT image to the two X-ray images $(4 / 5 \mathrm{~mm}$

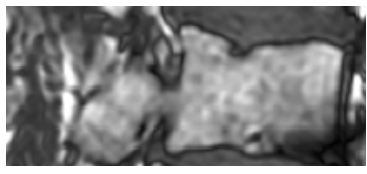

(a)

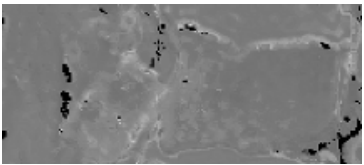

(d)

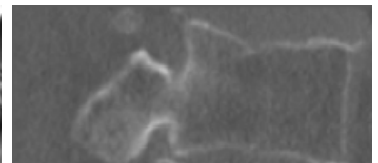

(b)

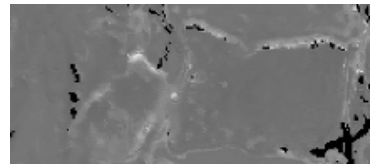

(e)

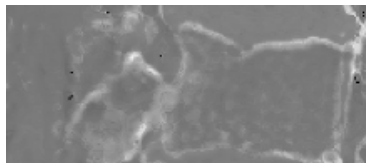

(c)

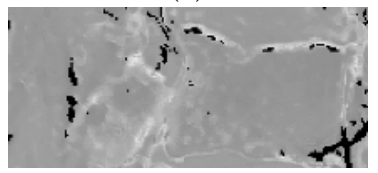

(f)

Fig. 2. The same slices of vertebra L3 of subject A: a) BFFE; b) transformed CT; c) trained on SA-L3; d) trained on SB-L3; e) trained on SB-L1; f) trained on SB-L1,L3 
Table 2. The $100 \%$ and $90 \%$ capture ranges and accuracy of successful $(<2 \mathrm{~mm}$ mTRE) registrations within the capture range for $2 \mathrm{D}-3 \mathrm{D}$ registration using a gradientbased method on 3DRX data, CT data, transformed/resampled CT data ('trans CT'), and four generated CT-like datasets of vertebra L3 of subject A.

\begin{tabular}{lccc}
\hline \hline & \multicolumn{3}{c}{ Gradient-based method } \\
& $100 \%(\mathrm{~mm})$ & $90 \%(\mathrm{~mm})$ & Err. $(\mathrm{mm})$ \\
\hline \hline 3DRX & 5 & 6 & 0.30 \\
$\mathrm{CT}$ & 4 & 5 & 0.22 \\
trans CT & 3 & 5 & 0.34 \\
\hline train SA-L3 & 3 & 5 & 0.46 \\
train SB-L3 & 2 & 4 & 0.55 \\
train SB-L1 & 3 & 4 & 0.45 \\
train SB-L1/L3 & 3 & 4 & 0.61 \\
\hline MR (BFFE) & 1 & 1 & 0.81 \\
\hline \hline
\end{tabular}

with mTRE of $0.22 \mathrm{~mm}$ ). Registration of the transformed and resampled CT image, i.e. the image used when training the LUT, had a slightly smaller capture range $(3 / 5 \mathrm{~mm}$ with $\mathrm{mTRE}$ of $0.34 \mathrm{~mm})$. Registration performance for the four generated CT-like datasets (approximately $3 / 4 \mathrm{~mm}$ and $\mathrm{mTRE}$ of $0.52 \mathrm{~mm}$ ) was almost equal to the performance of the transformed CT image. As it can be assumed that the results obtained for the transformed CT image are the best attainable for the CT-like images, since the LUT is trained from the transformed CT image, this indicates the potential of the method. Furthermore, it seemed that the training set used to define the mapping function from multispectral $\mathrm{MR}$ intensities to $\mathrm{CT}$ values did not have a large influence on the result. The performance of MR to X-ray registration using the CT-like data was much better than when using gradient-based registration directly (after windowing and optimizing parameters for relevant point extraction) on MR data (1/1 mm and mTRE of $0.81 \mathrm{~mm}$ ) and better than the results reported in literature [5]. This may be explained by numerous points extracted on soft-tissue boundaries in the original MR dataset which introduce boundaries that are not clearly visible in the X-ray images and thus make gradient-based registration applied directly on MR data difficult.

The current study has several limitations. First, we used a limited number of training and evaluation data. Second, the quality of the data was limited, and therefore we should be cautious in generalizing the results. It is subject of further investigation whether the result will be the same when a larger dataset is used and when LUTs are trained and evaluated on more subjects. Moreover, although it is difficult to acquire training data in vivo, this may be required for in vivo application, as we observed considerable differences in the histograms of MR data between healthy subjects and in the postmortem scans.

Furthermore, in our experiment a simple mapping function, i.e. the median, was used for generating the LUT. Other mappings of CT intensities can be 
investigated as well. Possible extensions are to take neighborhood information or other spatial information into account, or using extra features, e.g. gradient and texture information or intensity profiles. Another option is to use the variance of the occurring CT values for a specific MR intensity triplet, for example to determine the confidence when generating the CT-like image. It can also be investigated if less than three MR images or other MR protocols can be used to achieve similar results.

Finally, the performance of other types of 2D-3D registration methods, such as feature-based and intensity-based methods, using generated CT-like data should be investigated. By using a CT-like image, intensity-based methods can potentially overcome their current limitation in registering MR data to X-ray images.

\section{Conclusions}

A method has been presented to simulate CT data from multispectral MR data so as to allow indirect MR to X-ray registration. In preliminary experiments, it was shown that gradient-based registration of the CT-like data to X-ray data yielded results that approximated the accuracy of original transformed CT data to X-ray registration, and that it outperformed registration directly on the MR data.

Acknowledgment. We thank the Netherlands Organization for Scientific Research (NWO) for providing a travel grant.

\section{References}

1. Hipwell, J., Penney, G., McLaughlin, R., et al.: Intensity-based 2-D-3-D registration of cerebral angiograms. IEEE Trans. Med. Imag. 22 (2003) 1417-1426

2. Feldmar, J., Malandain, G., Ayache, N., et al.: Matching 3D MR angiography data and 2D X-ray angiograms. In: Proceedings of CVRMed-MRCAS '97. (1997) 129-138

3. Liu, A., Bulitt, E., Pizer, S.: 3D/2D registration via skeletal near projective invariance in tubular objects. In: MICCAI. (1998) 952-963

4. Rohlfing, T., Maurer Jr., C.: A novel image similarity measure for registration of 3-D MR images and X-ray projection images. In: MICCAI, Part 2. (2002) 469-476

5. Tomaževič, D., Likar, B., Slivnik, T., et al.: 3-D/2-D registration of CT and MR to X-ray images. IEEE Trans. Med. Imag. 22 (2003) 1407-1416

6. van de Kraats, E., Penney, G., Tomaževič, D., et al.: Standardized evaluation of 2D-3D registration. In: MICCAI, Part 1. (2004) 574-581 